Cite this: Phys. Chem. Chem. Phys., 2014, 16, 15910

Received 22nd April 2014, Accepted 16th June 2014 DOI: $10.1039 / c 4 c p 01741 b$

www.rsc.org/pccp

\title{
Hydrogen bonding of nitroxide spin labels in membrane proteins
}

\author{
P. Gast, ${ }^{* a}$ R. T. L. Herbonnet, ${ }^{a}$ J. Klare, ${ }^{b}$ A. Nalepa, ${ }^{c}$ C. Rickert, ${ }^{b}$ D. Stellinga, ${ }^{a}$ \\ L. Urban, ${ }^{b}$ K. Möbius, ${ }^{\text {cd }}$ A. Savitsky, ${ }^{c}$ H.-J. Steinhoff ${ }^{b}$ and E. J. J. Groenen ${ }^{a}$
}

\begin{abstract}
On the basis of experiments at $275 \mathrm{GHz}$, we reconsider the dependence of the continuous-wave EPR spectra of nitroxide spin-labeled protein sites in sensory- and bacteriorhodopsin on the micro-environment. The high magnetic field provides the resolution necessary to disentangle the effects of hydrogen bonding and polarity. In the $g_{x x}$ region of the $275 \mathrm{GHz}$ EPR spectrum, bands are resolved that derive from spin-label populations carrying no, one or two hydrogen bonds. The $g_{x x}$ value of each population varies hardly from site to site, significantly less than deduced previously from studies at lower microwave frequencies. The fractions of the populations vary strongly, which provides a consistent description of the variation of the average $g_{x x}$ and the average nitrogen-hyperfine interaction $A_{z z}$ from site to site. These variations reflect the difference in the proticity of the micro-environment, and differences in polarity contribute marginally. Concomitant W-band ELDOR-detected NMR experiments on the corresponding nitroxide in perdeuterated water resolve population-specific nitrogen-hyperfine bands, which underlies the interpretation for the proteins.
\end{abstract}

\section{Introduction}

Site-directed spin labeling using nitroxide radicals has opened up the possibility to probe the local polarity and proticity in a protein with continuous-wave electron paramagnetic resonance (EPR). In frozen solution, the EPR spectra reveal the $g$-tensor and the nitrogen-hyperfine tensor, which are both sensitive to the micro-environment of the nitroxide label. Such an approach has been successfully applied to various membrane and water soluble proteins, ${ }^{1,2}$ and the variation of the EPR parameters from site to site was analyzed in terms of the effects of local polarity and of hydrogen bonding. In particular, the $A_{z z}$ component of the nitrogen-hyperfine tensor (in the coordinate system with the $x$ axis along the $\mathrm{N}-\mathrm{O}$ bond and the $z$ axis perpendicular to the nitroxide ring plane) has been argued to present a measure of the polarity and the behaviour of $g_{x x}$ versus $A_{z z}$ to reflect the interplay of polarity and proticity, where the latter represents the propensity to form hydrogen bonds. Recently, pulsed and continuous-wave EPR were combined to disentangle the sources of protons involved in hydrogen bonding. ${ }^{3}$

\footnotetext{
${ }^{a}$ Huygens-Kamerlingh Onnes Laboratory, Department of Physics, Leiden University, P.O. Box 9504, NL-2300 RA Leiden, The Netherlands.

E-mail: gast@physics.leidenuniv.nl

${ }^{b}$ Department of Physics, University of Osnabrück, Barbarastrasse 7 , D-49076 Osnabrück, Germany.E-mail: hsteinho@uni-osnabrueck.de ${ }^{c}$ Max Planck Institute for Chemical Energy Conversion, Stiftstrasse 34-36, D-45470 Mülheim an der Ruhr, Germany. E-mail: anton.savitsky@cec.mpg.de

${ }^{d}$ Department of Physics, Free University Berlin, Arnimallee 14, D-14195 Berlin, Germany.E-mail: moebius@physik.fu-berlin.de
}

For nitroxide radicals, Lebedev and co-workers ${ }^{4}$ noticed by high-field EPR already in the early 1980's a linear correlation between $g_{x x}$ and $A_{z z}$, where $g_{x x}$ decreases and $A_{z z}$ increases with increasing solvent polarity. The influence of hydrogen bonding on $g_{x x}$ has been referred to first for five-membered ring nitroxide spin labels ${ }^{5}$ and on $A_{z z}$ for the perdeuterated six-membered ring nitroxide spin label tempone in perdeuterated ethanol. ${ }^{6}$ Polarity effects on the EPR parameters are small at higher dielectric constants in aqueous environments where the effect of hydrogen bonds was found to become dominant. ${ }^{7}$ These observations were theoretically analyzed by semi-empirical molecular orbital methods, ${ }^{8}$ by density-functional-theory calculations, ${ }^{7}$ and by unrestricted Hartree-Fock calculations. ${ }^{9}$ Trends were reproduced, but the contribution of polarity and of hydrogen bonding to the observed variation of the EPR parameters remained undecided.

At X-band $(9.5 \mathrm{GHz})$ microwave frequency, values of $g_{z z}$ and $A_{z z}$ of nitroxides can be obtained from rigid-limit spectra. At W-band ( $95 \mathrm{GHz}$ ) microwave frequency, all $g$-tensor components become resolved. The $g_{x x}$ region of the EPR spectra of nitroxides in frozen polar/protic solution shows, besides nitrogen hyperfine/ quadrupole structure, different components related to distinct local environments. ${ }^{10}$ Corresponding differences in the nitrogenhyperfine coupling $A_{z z}$ are expected, but remain hidden in the broad EPR lines. Recently, distinct $A_{z z}$ contributions were observed in ELDOR-detected NMR (EDNMR) experiments on a pyrroline-type nitroxide radical in 2-propanol, and described in terms of nitroxides in different situations of hydrogen bonding. ${ }^{11}$

For nitroxide-labeled proteins the hyperfine structure is no longer resolved in the $g_{x x}$ region of the EPR spectra. Instead, for 
such systems the $g_{x x}$ line at W-band reveals a complex structure. ${ }^{12}$ In an extensive study, nitroxide spin labels covalently attached to many sites in both membrane and water-soluble proteins were investigated, mostly at W-band. ${ }^{2}$ A consistent description of the $g_{x x}$ region of the EPR spectra was obtained in terms of the contributions of three populations characterized by distinct $g_{x x}$ values, $g_{x x}^{i}(i=0,1,2)$, for each investigated position of the spin label at the protein. The $g_{x x}^{i}$ values and the fractions of the different spin-label populations were correlated to the average hyperfine splitting $\left\langle A_{z z}\right\rangle$ and the populations were discussed in terms of hydrogen bonding to the nitroxide moiety. In this study, a few experiments at 275 and $360 \mathrm{GHz}$ were included, which clearly showed that a further increase of the Zeeman resolution would facilitate the interpretation of the sub-structure in the $g_{x x}$ region of the spectrum.

Here we benefit from the higher resolution at $275 \mathrm{GHz}$ and present the results of an EPR study at this microwave frequency of a number of spin label sites in the membrane proteins sensory rhodopsin and bacteriorhodopsin. Supporting data are reported for the deuterated nitroxide radical 3-hydroxymethyl-2,2,5,5tetramethylpyrrolin-1-oxyl $\left(\mathrm{R} 1-\mathrm{D}_{16}\right)$ dissolved in $\mathrm{D}_{2} \mathrm{O}$. Analysis of the variation of $g_{x x}^{i}$, of the average $g_{x x}$, and of the average $A_{z z}$ with the protein site reveals the dominant role of hydrogen bonding.

\section{Materials and methods}

\section{Sample preparation}

Protein expressions, spin labeling and sample preparation by reconstitution in purple membrane lipids were carried out as described previously. ${ }^{13,14}$ The spin label (1-oxyl-2,2,5,5-tetramethylpyrroline-3-methyl)methanethiosulphonate (MTSSL; TRC, Toronto) was covalently attached to the cysteine residues of the protein cysteine exchange mutants according to the method described in ref. 14. The proteins contained spin labels at the following positions: sensory rhodopsin (sr): sr16, sr17, sr34, sr36, sr89, sr90, sr95 and sr136, and bacteriorhodopsin (br): br129 and br166. The samples were concentrated by either first concentrating the solution in an Eppendorf centrifuge or concentrating the sample inside the EPR sample tube using a table top swing-out centrifuge.

The perdeuterated nitroxide spin probe R1- $\mathrm{D}_{16}$ used in this study was synthesized as described previously. ${ }^{10}$ The solvents $\mathrm{D}_{2} \mathrm{O}$ and glycerol- $\mathrm{D}_{8}$ were obtained from Deutero $\mathrm{GmbH}$ and Sigma Aldrich, respectively.

\section{EPR at $275 \mathrm{GHz}$}

The $275 \mathrm{GHz}$ continuous-wave (cw) EPR and pulsed electron-nuclear double resonance (ENDOR) spectra have been recorded using a home-built spectrometer operating at $275.7 \mathrm{GHz} .{ }^{15}$ The spectrometer is equipped with a $\mathrm{TE}_{011}$ cylindrical cavity with a diameter of $1.4 \mathrm{~mm}$. The quartz sample tubes (VitroCom Inc.) have an outer diameter of $0.25 \mathrm{~mm}$ and an inner diameter of $0.15 \mathrm{~mm}$. The effective sample volume inside the cavity is approximately $27 \mathrm{nl}$.

All cw spectra have been measured at a temperature of $40 \mathrm{~K}$. The modulation frequency was $1.380 \mathrm{kHz}$, and the modulation amplitude approximately $0.4 \mathrm{mT}(0.12 \mathrm{mT}$ for the deuterated sample). The microwave power of $1 \mathrm{~mW}$ was attenuated by 36.5 to $40 \mathrm{~dB}$. The scans were all performed over the same field range $(9.7950 \mathrm{~T}$ to $9.8550 \mathrm{~T})$, at the same scan rate $\left(0.12 \mathrm{mT} \mathrm{s}^{-1}\right)$, and with 2000 data points.

The inner chamber of the cryostat, holding the probe head, was deoxygenated by pumping out the air at a temperature of about $120-160 \mathrm{~K}$ and filling with helium gas.

Calibration of the magnetic field sensed by the EPR sample was accomplished by measuring the proton Larmor frequency in a Mims-type ${ }^{1} \mathrm{H}$-ENDOR experiment at $9.8200 \mathrm{~T}$ and $5 \mathrm{~K}$. The measured EPR spectra were subsequently corrected for the difference between the calculated magnetic-field value and the set value of $9.8200 \mathrm{~T}$. The correction varied between 0.8 and $1.5 \mathrm{mT}$. For the deuterated nitroxide in deuterated solvent, the calibration of the magnetic field was based on $g_{z z}=2.00221$.

For the determination of the three $g_{x x}^{i}$ values only the data points between $9.8000 \mathrm{~T}$ and $9.8150 \mathrm{~T}$ were used. The EPR spectra contained in some cases a minor contribution of dispersion and of Mn(II) signals. This made a simulation of the entire EPR spectrum less accurate in the $g_{x x}$ region than a fit of only this part of the spectrum. The fits of the EPR spectra in the $g_{x x}$ region with four Gaussians were obtained using OriginPro 7.5. Three of the Gaussians represent the individual components $g_{x x}^{0}, g_{x x}^{1}$ and $g_{x x}^{2}$, while the tail of a fourth is used as a baseline to compensate for the residual contribution of the rest of the spectrum, most notably the $g_{y y}$ peak. The starting parameters (line width, field position, intensity and offset) for these " $3+1$ " Gaussian fits were left unrestricted. The peak positions of the Gaussians are then used to calculate the $g_{x x}^{i}$ values and the area under each Gaussian as a measure of the fraction of each population. Error margins for the measured $g_{x x}^{i}$ values were determined from fits of EPR spectra, which were recorded twice. Before fitting the $g_{x x}$ region a linear baseline correction was executed and the whole spectrum was shifted along the magnetic field axis according to the results from the ${ }^{1} \mathrm{H}$-ENDOR experiment.

\section{W-band EPR}

The W-band cw EPR measurements have been performed at $80 \mathrm{~K}$ with a home-built spectrometer operating at a microwave frequency of $95 \mathrm{GHz} .{ }^{16}$ The magnetic field was modulated at $8 \mathrm{kHz}$ with an amplitude of $30 \mu \mathrm{T}$.

The W-band EDNMR experiments have been performed on a modified Bruker Elexsys E680 spectrometer operating at about $94 \mathrm{GHz} .{ }^{11}$ The EDNMR experiments made use of a two-pulse Hahn-echo detection at microwave frequency $\nu_{1}$, matched to the cavity resonance. The high-turning angle (HTA) microwave pulse was applied at the microwave frequency $\nu_{2}$. The spectra were acquired by continuously sweeping $\nu_{2}$ at a fixed magnetic field, in steps of $70 \mathrm{kHz}$. The length and amplitude of the HTA pulse were adjusted to obtain an ELDOR $\pi$-pulse on the forbidden transition of interest at the $g_{z z}, m_{\mathrm{I}}=-1$ field position. The HTA pulse length was set to $2.5 \mu \mathrm{s}$ at a $2.6 \times 10^{7}$ rad per s pulse amplitude. The detection sequence was set $7 \mu$ s after the HTA pulse to ensure the decay of electron-spin coherencies. 
The length of the detection $\pi / 2$ pulse was set to $100 \mathrm{~ns}$, and the pulse separation to $1 \mu \mathrm{s}$. The echo was integrated over $1.4 \mu \mathrm{s}$ around its maximum for optimal resolution. For the twodimensional EDNMR data surface, EDNMR traces were recorded in the field range of $1.2 \mathrm{mT}$ around the $g_{z z}, m_{\mathrm{I}}=-1$ spectral field position. A measurement repetition rate of $500 \mathrm{~Hz}$ was used to allow the echo signal to restore between consecutive pulse trains. The EDNMR data were analysed according to the procedure reported previously. ${ }^{11}$

\section{X-band EPR}

The X-band cw EPR measurements have been performed at $160 \mathrm{~K}$ with a home-built spectrometer equipped with an AEG $\mathrm{H} 103$ rectangular cavity. The microwave power was set to $0.2 \mathrm{~mW}$ and the magnetic field modulated with an amplitude of $0.25 \mathrm{mT}$. A B-NM 12 B-field meter (Bruker) was employed to measure the magnetic field and a flow cryostat (Oxford ESR $900)$ to stabilize the sample temperature. Sample volumes of $40 \mu \mathrm{l}$ at protein concentrations of $15-20 \mathrm{mg} \mathrm{ml}^{-1}$ were loaded into EPR quartz capillaries of $3 \mathrm{~mm}$ inner diameter.

The $A_{z z}$ values were determined by fitting simulated spectra to the experimental ones using the program DIPFIT, ${ }^{17}$ with an estimated error of $\pm 0.01 \mathrm{mT}$. These values are more accurate than corresponding values determined from $\mathrm{cw}$ spectra at higher microwave frequencies.

\section{Results and discussion}

Fig. 1a shows the cw EPR spectrum at $275 \mathrm{GHz}$ and $40 \mathrm{~K}$ of sensory rhodopsin spin-labeled at position 90, which is a typical example of the spectra that we have obtained for all ten systems. The spectrum reflects a nitroxide powder spectrum in which the principal $g$ regions are fully resolved owing to the high microwave frequency and correspondingly high magnetic field. The $g_{z z}$ region, around $9.838 \mathrm{~T}$, shows three nitrogen-hyperfine transitions split by $A_{z z}$. Some of the spectra contain small spurious signals in this region at 9.830, 9.839 and $9.848 \mathrm{~T}$, which derive from the presence of $\mathrm{Mn}$ (II). The $g_{x x}$ region of the spectrum, between 9.800 and $9.815 \mathrm{~T}$, clearly shows structure. In Fig. 1b, this spectral region is represented for all samples, from top to bottom in the order of increasing nitrogen-hyperfine splitting. In most spectra three peaks can be discerned. As outlined in Materials and methods and illustrated for sr90 in the inset of Fig. 1a, a Gaussian fit was performed of the $g_{x x}$ region of the spectra. The results of these fits in terms of the distinct contributions (fractions $\mathrm{fr}^{0}, \mathrm{fr}^{1}$, and $\mathrm{fr}^{2}$ ) of three $g_{x x}$-tensor components $\left(g_{x x}^{0}, g_{x x}^{1}\right.$ and $\left.g_{x x}^{2}\right)$ are summarized in Table 1.

The observation of sub-structure in the $g_{x x}$ region, which does not derive from nitrogen-hyperfine or quadrupole splitting, is consistent with EPR spectra at lower microwave frequency. In a study of about 100 spin-labeled sites in various proteins at W-band, ${ }^{2}$ the EPR spectra were simulated as the sum of three components characterized by different $g_{x x}$ values. The three components were interpreted as nitroxide populations carrying no hydrogen bond, one hydrogen bond and two hydrogen bonds. The presence of different populations was clear from the W-band experiments, but the strong overlap of the EPR lines limited the accuracy of the determination of the corresponding $g_{x x}^{i}$ values.

The improved $g$-resolution of the EPR spectra at $275 \mathrm{GHz}$ confirms the presence of three nitroxide populations for the spin-labeled protein sites and allows for a more precise analysis. We find $g_{x x}$ values in narrow ranges: $g_{x x}^{0}$ between 2.00913 and 2.00922, $g_{x x}^{1}$ between 2.00846 and 2.00864, and $g_{x x}^{2}$ between 2.00801 and 2.00825 (Table 1). The difference between $g_{x x}^{0}$ and $g_{x x}^{1}$ is on average $640 \mathrm{ppm}$ and between $g_{x x}^{1}$ and $g_{x x}^{2}$ is on average $420 \mathrm{ppm}$. These differences agree with those theoretically predicted for the change of $g_{x x}$ upon formation of one and two hydrogen bonds to a nitroxide, which corroborates the assignment of the populations. $^{7,8,18}$

Fig. 2 shows a plot of the $g_{x x}^{i}$ values of the protein sites as a function of the $z$ component of the nitrogen-hyperfine splitting, the latter being obtained from X-band cw EPR spectra. We label

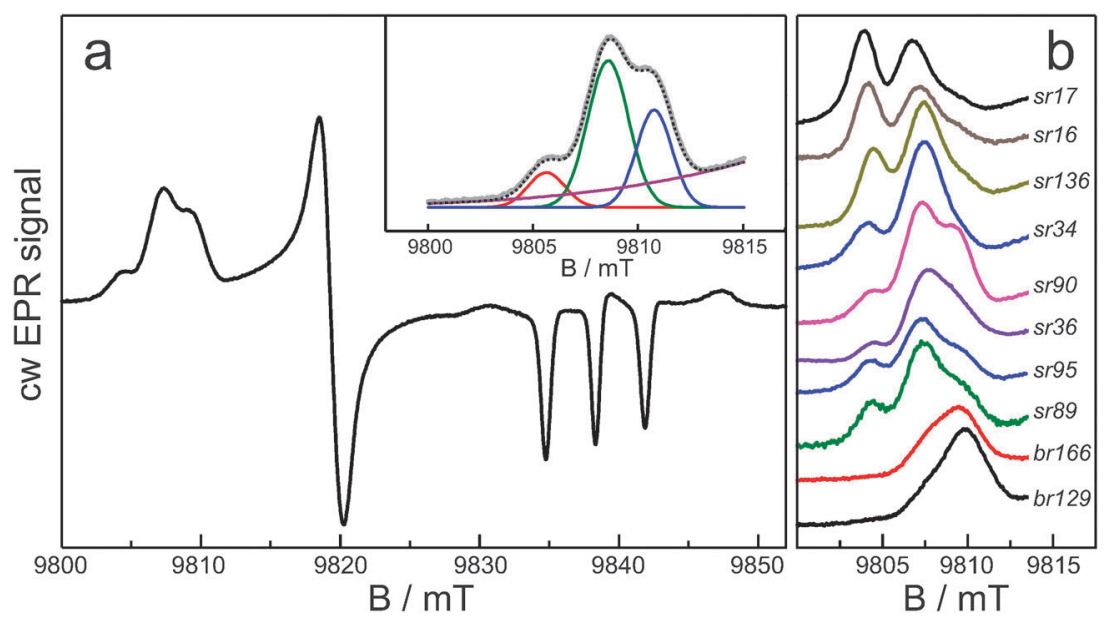

Fig. 1 Continuous-wave EPR spectra at $275 \mathrm{GHz}$ and $40 \mathrm{~K}$ of spin-labeled proteins. (a) Full spectrum for sensory rhodopsin spin-labeled at position 90 ; the inset shows the result of the Gaussian fit of the $g_{x x}$ region of this spectrum. (b) The $g_{x x}$ region of the spectra of all spin-labeled proteins in this study. 
Table 1 Summary of data for the spin-labeled protein sites. The values of $g_{x x}^{i}$ and of $\operatorname{fr}^{i}(i=0,1,2)$ are derived from the Gaussian fit of the $g_{x x}$ region of the cw EPR spectra at $275 \mathrm{GHz}$. The $\left\langle g_{x x}^{i}\right\rangle$ values concern the average of $g_{x x}^{i}$ over the ten protein sites. The values of $\left\langle A_{z z}\right\rangle$ are derived from cw EPR spectra at X-band. The values of $\left\langle g_{x x}\right\rangle$ correspond to the first moment of the $g_{x x}$ region of the cW EPR spectra at $275 \mathrm{GHz}$, the values of $\left\langle g_{x x}\right\rangle^{\mathrm{C}}$ correspond to $\mathrm{fr}^{0} \cdot g_{x x}^{0}+\mathrm{fr}^{1} \cdot g_{x x}^{1}+\mathrm{fr}^{2} \cdot g_{x x}^{2}$. The $g$ values are presented as deviations from $g=2$ in units of $10^{-4}$

Spin-labeled

\begin{tabular}{llllllllll} 
protein & $g_{x x}^{0}$ & $g_{x x}^{1}$ & $g_{x x}^{2}$ & $\mathrm{fr}^{0}$ & $\mathrm{fr}^{1}$ & $\mathrm{fr}^{2}$ & $\left\langle A_{z z}\right\rangle / \mathrm{mT}$ & $\left\langle g_{x x}\right\rangle$ & $\left\langle g_{x x}\right\rangle^{\mathrm{C}}$ \\
\hline sr17 & 92.2 & 86.4 & 82.5 & 0.48 & 0.34 & 0.18 & 3.42 & 88.9 & 88.5 \\
sr16 & 91.9 & 85.8 & 81.3 & 0.39 & 0.48 & 0.14 & 3.43 & 87.6 & 87.6 \\
sr136 & 91.3 & 85.3 & 80.8 & 0.30 & 0.58 & 0.12 & 3.49 & 86.5 & 86.6 \\
sr34 & 92.2 & 85.2 & 80.6 & 0.21 & 0.72 & 0.07 & 3.54 & 86.6 & 86.4 \\
sr90 & 91.6 & 85.6 & 81.1 & 0.10 & 0.55 & 0.35 & 3.55 & 84.9 & 84.6 \\
sr36 & 91.4 & 85.0 & 80.9 & 0.07 & 0.60 & 0.33 & 3.55 & 84.1 & 84.1 \\
sr95 & 91.8 & 85.6 & 81.0 & 0.19 & 0.58 & 0.23 & 3.58 & 85.6 & 85.7 \\
sr89 & 91.5 & 85.4 & 80.7 & 0.19 & 0.53 & 0.28 & 3.63 & 85.2 & 85.2 \\
br166 & - & 84.6 & 80.6 & 0 & 0.34 & 0.66 & 3.64 & 82.0 & 82.0 \\
br129 & - & 85.0 & 80.15 & 0 & 0.16 & 0.84 & 3.67 & 80.9 & 81.0 \\
$\left\langle g_{x x}^{i}\right\rangle$ & 91.7 & 85.3 & 81.1 & & & & & &
\end{tabular}

the splitting by $\left\langle A_{z z}\right\rangle$, anticipating that the observed value of $A_{z z}$ can be the average of distinct $A_{z z}$ values, each associated with one of the $g_{x x}$ components. The $A_{z z}$ peaks in the EPR spectra show no structure, which means that distinct hyperfine transitions, if present, are hidden in the inhomogeneously broadened line. The linear fits in Fig. 2 correspond to small slopes, from $-0.17 \mathrm{~T}^{-1}$ for the spin label carrying no hydrogen bond to $-0.52 \mathrm{~T}^{-1}$ for the spin label carrying two hydrogen bonds. For $\left\langle A_{z z}\right\rangle$ values between 3.43 and $3.63 \mathrm{mT}$, the variation of $g_{x x}^{i}(i=0$, $1,2)$ is even negligible, taken into account the accuracy of $10 \mathrm{ppm}$. The slopes are significantly smaller than the slopes of -1.2 to $-1.6 \mathrm{~T}^{-1}$ derived from similar EPR experiments at W-band. ${ }^{2}$ The difference results from the limitation in the determination of $g_{x x}^{i}$ at the lower microwave frequency due to the fact that the decomposition of the EPR spectra at W-band is less accurate. The few data at 275 and $360 \mathrm{GHz}$ in ref. 2 are fully consistent with the present findings. The slopes derived from the $275 \mathrm{GHz}$ data are at least an order of magnitude smaller

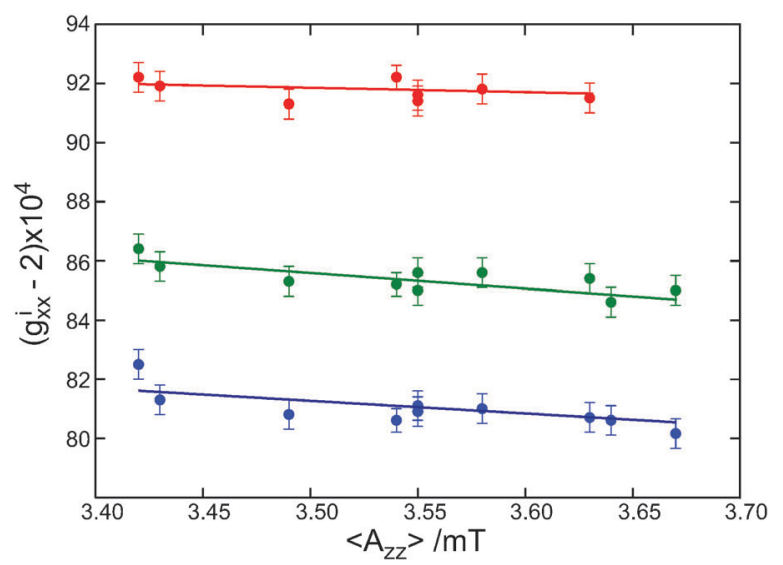

Fig. 2 The variation of $g_{x x}^{i}(i=0$ red, $i=1$ green and $i=2$ blue $)$ as a function of $\left\langle A_{z z}\right\rangle$ for all spin-labeled proteins. than the value of -5.1 and $-6.5 \mathrm{~T}^{-1}$ predicted theoretically for nitroxides in aprotic and protic solvents. ${ }^{8}$ The observed slopes are also smaller than the value of $-0.8 \mathrm{~T}^{-1}$, which was obtained from measurements of the unbound nitroxide spin label MTSSL in solvents of different polarity. ${ }^{7}$ Apparently, polarity is not decisive as regards the variation of $g_{x x}^{i}$ with $\left\langle A_{z z}\right\rangle$. This conclusion needs further discussion after consideration of the factors that determine $\left\langle A_{z z}\right\rangle$.

The nitrogen-hyperfine lines at $g_{z z}$ do not show structure, but, as seen in Fig. 1a, the outer lines corresponding to $m_{\mathrm{I}}= \pm 1$ are broader than the central line corresponding to $m_{\mathrm{I}}=0$ (FWHM $0.80 \mathrm{mT}$ vs. $0.69 \mathrm{mT}$ ). This difference could derive from a distribution of $A_{z z}$ values, but the observation of distinct $g_{x x}$ populations rather suggests distinct $A_{z z}$ values. This interpretation is supported by EPR experiments on the nitroxide radical $\mathrm{R} 1$. This radical is relevant because the methanethiosulphonate substituted derivative of R1 corresponds to the spin label of the proteins. We have investigated $\mathrm{R} 1-\mathrm{D}_{16}$ in $\mathrm{D}_{2} \mathrm{O}$ with $5 \%$ and $10 \%(\mathrm{v} / \mathrm{v})$ of glycerol- $\mathrm{D}_{8}$, which concerns an aqueous micro-environment of the nitroxide radical and in that sense can be considered a limiting case in relation to the spin-label sites in the proteins. Fig. 3a shows the high-field cw EPR spectrum of the nitroxide radical at $275 \mathrm{GHz}$ and, for comparison, that of bacteriorhodopsin labeled at position 129 (br129). The spectra are indeed similar. The R1- $\mathrm{D}_{16}$ spectra in $\mathrm{D}_{2} \mathrm{O}$ containing $5 \%$ or $10 \%(\mathrm{v} / \mathrm{v})$ of glycerol- $\mathrm{D}_{8}$ are identical, which indicates the dominant interaction of the nitroxide radical with water molecules. The $g_{x x}$ region of the nitroxide spectrum reveals two spectral contributions with different $g$-tensor principal values: $g_{x x}^{1}=2.00831 \pm 0.00004$ and $g_{x x}^{2}=2.00790 \pm$ 0.00004 . The two contributions in the $g_{x x}$ region can also be distinguished in the cw EPR spectrum at W-band (Fig. 3b). The overall shape of the $g_{x x}$ band at W-band is different from that at $275 \mathrm{GHz}$ because of nitrogen-hyperfine/quadrupole structure, which is still partly resolved at the lower microwave frequency. ${ }^{10}$ The two contributions are ascribed to two fractions of the nitroxide radical, which forms either one (population 1) or two (population 2) hydrogen bonds with the water molecules. The difference of $400 \mathrm{ppm}$ in the $g$-values is virtually identical to the difference observed for the corresponding singly and doubly hydrogen-bonded populations of the spinlabeled sites in the proteins. As for the spin-labeled proteins, the $g_{z z}, m_{\mathrm{I}}= \pm 1$ EPR lines are broader than the $g_{z z}, m_{\mathrm{I}}=0$ line (FWHM $0.45 \mathrm{mT} v s$. $0.32 \mathrm{mT}$ at $275 \mathrm{GHz}$ ), and different $A_{z z}$ values of the two populations are not resolved. Here the high resolution of EDNMR spectroscopy comes to help.

Fig. 4 shows the W-band EDNMR spectra of $\mathrm{R} 1-\mathrm{D}_{16}$ in $\mathrm{D}_{2} \mathrm{O}$ with $5 \%(\mathrm{v} / \mathrm{v})$ glycerol- $\mathrm{D}_{8}$. In the $g_{z z}, m_{\mathrm{I}}=-1$ spectral region, the lines originating from forbidden single-quantum transitions, $\left|m_{\mathrm{S}}=-1 / 2, m_{\mathrm{I}}=-1\right\rangle \rightarrow|1 / 2,0\rangle$ and $|-1 / 2,0\rangle \rightarrow|1 / 2,-1\rangle$, are observed at positive frequencies $\Delta \nu=\nu_{2}-\nu_{1}$. At the $g_{z z}, m_{\mathrm{I}}=-1$ canonical field position, the lines are centered around the principal $A_{z z} / 2$ value. Instead of one pair of lines, however, two partially resolved pairs of lines of different intensities and positions are observed similar to the situation found previously for $\mathrm{R} 1-\mathrm{D}_{16}$ in 2-propanol. ${ }^{11}$ These pairs are assigned to two 

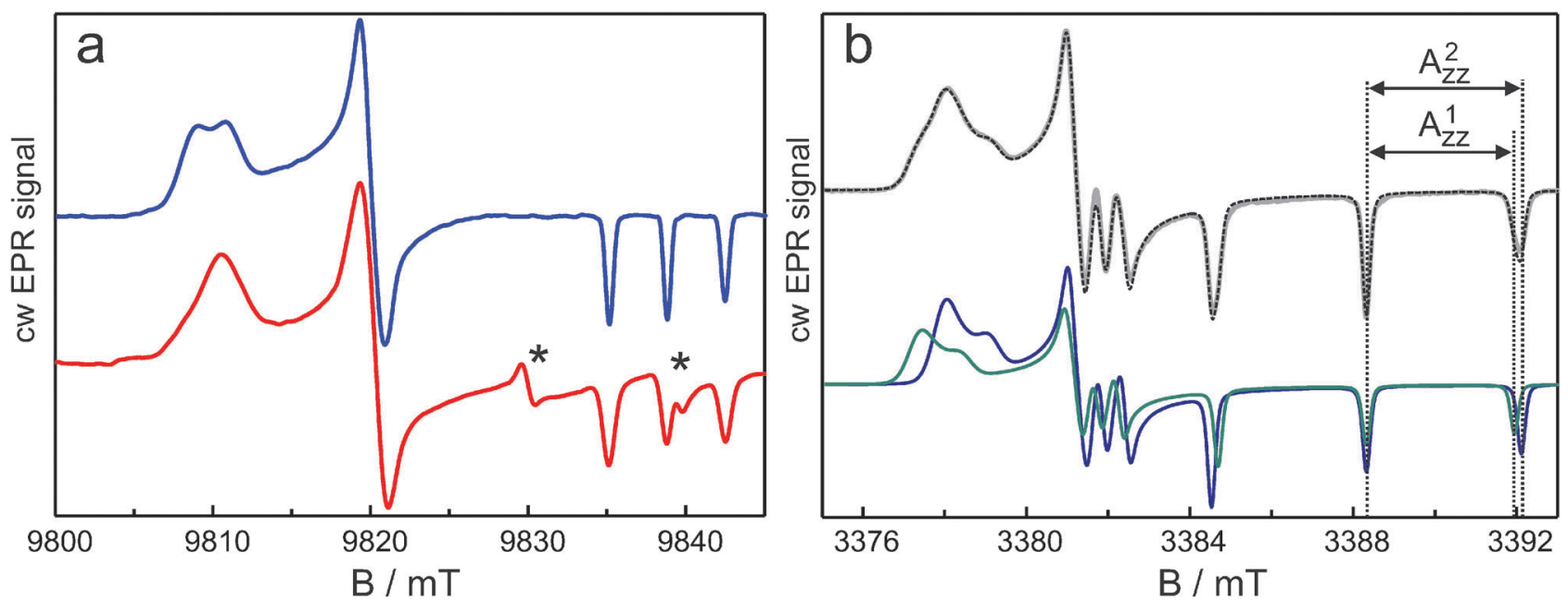

Fig. 3 Continuous-wave EPR spectra: (a) at $275 \mathrm{GHz}$ and $40 \mathrm{~K}$ of $1 \mathrm{mM} \mathrm{R} 1-\mathrm{D}_{16}$ in $\mathrm{D}_{2} \mathrm{O}-5$ vol\% glycerol- $\mathrm{D}_{8}$ (top) and of br129 (bottom), (b) at W-band $(94.95 \mathrm{GHz})$ and $80 \mathrm{~K}$ of $1 \mathrm{mM} \mathrm{R} 1-\mathrm{D}_{16}$ in $\mathrm{D}_{2} \mathrm{O}-5$ vol\% glycerol- $\mathrm{D}_{8}$. The spectra at the top in (b) concern the experimental spectrum (gray line) overlaid with the calculated spectrum (dashed line) obtained by numerical solution of the spin Hamiltonian including the electron Zeeman, nuclear Zeeman, nitrogen-hyperfine and nitrogen-quadrupole interaction, making use of the experimentally determined EPR parameters and fractional weights of the two populations. The spectra at the bottom in (b) concern the calculated spectra of the individual populations. * indicates the location of two $\mathrm{Mn}^{2+}$ lines.
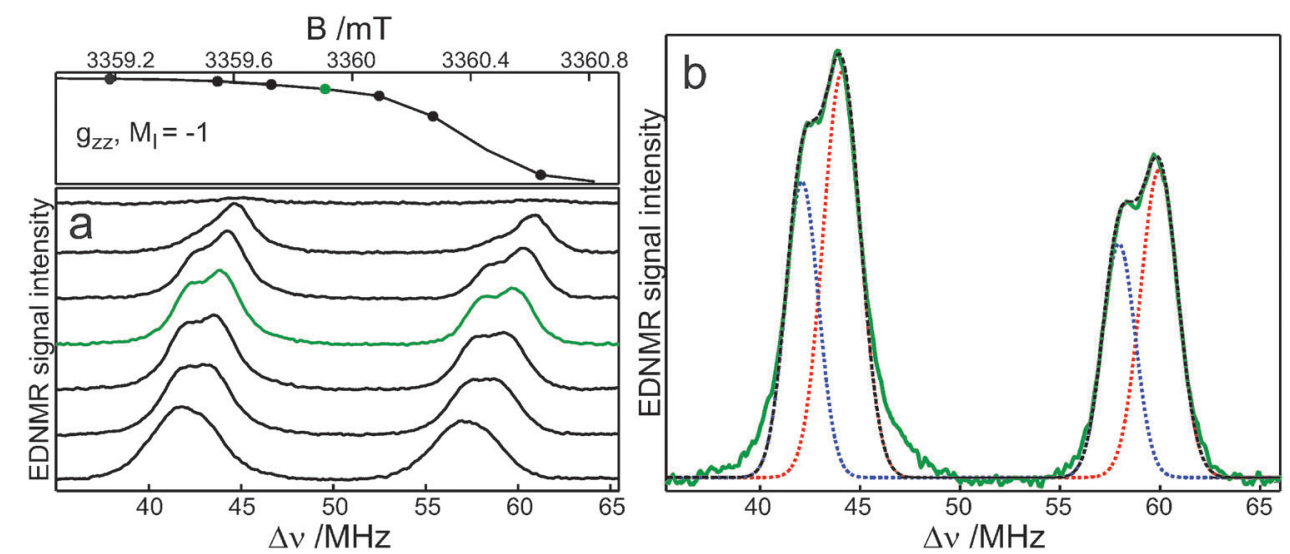

Fig. $4 \mathrm{~W}$-band $(94.02 \mathrm{GHz})$ EDNMR spectra of $1 \mathrm{mM} R 1-\mathrm{D}_{16}$ in $\mathrm{D}_{2} \mathrm{O}-5$ vol\% glycerol- $\mathrm{D}_{8}$ at $50 \mathrm{~K}$ acquired in the $g_{z z}, m_{1}=-1 \mathrm{spectral}$ region: (a) at the magnetic-field positions indicated by dots, (b) at the field position marked in green, including the best fit of this spectrum by two Gaussians.

nitroxide populations with distinct $A_{z z}$ values. The EDNMR recordings at each magnetic field in Fig. 4a have been analysed by using four Gaussian lines (for more details concerning the data analysis, see ref. 11). Fig. $4 \mathrm{~b}$ presents an example of such a fit. The analysis of the EDNMR spectrum yields $A_{z z}^{1}=101.6 \pm 0.1 \mathrm{MHz}(3.629 \pm$ $0.005 \mathrm{mT})$ and $A_{z z}^{2}=106.0 \pm 0.1 \mathrm{MHz}(3.786 \pm 0.005 \mathrm{mT})$ with spectral weights of the two nitroxide populations of $0.34 \pm 0.02$ and $0.66 \pm 0.02$, respectively. Additionally, the ${ }^{14} \mathrm{~N}$ quadrupole parameters, $P_{z z}$, have been obtained for the two sub-ensembles, $P_{z z}^{1}=$ $-1.54 \pm 0.05 \mathrm{MHz}$ and $P_{z z}^{2}=-1.49 \pm 0.05 \mathrm{MHz}$. The data obtained from the analysis of the EDNMR spectra quantitatively describe the CW EPR spectrum. Numerical calculation of the W-band cw EPR spectrum of R1-D ${ }_{16}$ as the sum of two spectra corresponding to two populations with their experimentally determined weights and EPR parameters ( $g$ values, nitrogen-hyperfine and quadrupole values, and an orientation dependent line width) yields a spectrum virtually identical to the experimental one (Fig. 3b).
The $A_{z z}^{1}$ and $P_{z z}^{1}$ values, which are assigned to the nitroxide radical hydrogen bonded to a single water molecule, are close to those observed for the hydrogen-bonded fraction of nitroxide radicals in 2-propanol $(99.6 \mathrm{MHz},-1.62 \mathrm{MHz})^{11}$ and glycerol (101.4 MHz, $-1.59 \mathrm{MHz}){ }^{10}$ The $A_{z z}^{2}$ value is significantly larger than in alcohols where a single hydrogen bond is formed ${ }^{10,11}$ and, thus, is assigned to the nitroxide radicals hydrogen bonded to two water molecules. This assignment is in agreement with the outcome of molecular-dynamics simulations, which predict the formation of one and preferentially two hydrogen bonds for a nitroxide spin probe in water, with a stepwise increase of $A_{z z}$ as compared to the bare nitroxide. ${ }^{19}$ Additionally, the quadrupole value $P_{z z}^{2}$ is lower than $P_{z z}^{1}$, supporting the assignment of the second fraction. ${ }^{10}$

As noticed above, also for the spin-labeled protein sites the $g_{z z}, m_{\mathrm{I}}= \pm 1$ lines are significantly broader than the $g_{z z}, m_{\mathrm{I}}=0$ line (Fig. 1). The observation of populations with distinct $A_{z z} / P_{z z}$ 

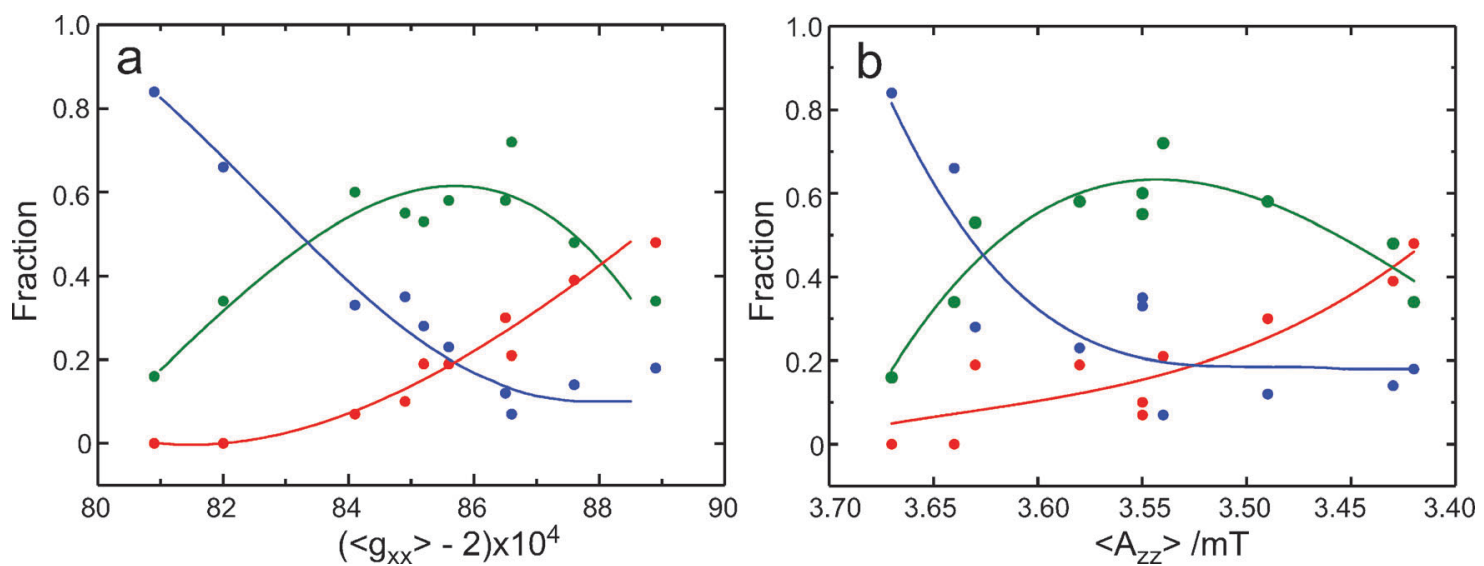

Fig. 5 Fractions ( $f r^{0}$ red, $\mathrm{fr}^{1}$ green, $\mathrm{fr}^{2}$ blue) as a function of $\left\langle g_{x x}\right\rangle$ (a) and of $\left\langle A_{z z}\right\rangle$ (b). The dots represent the experimental values of the fractions as determined from the analysis of the $g_{x x}$ region of the cw EPR spectra at $275 \mathrm{GHz}$. The lines only serve as a guide to the eye.

values for R1 in water suggests that also for the protein sites the broadening results from the overlap of lines corresponding to differently hydrogen-bonded species. We infer that both the sub-structure in the $g_{x x}$ region and the different width of the $A_{z z}$ lines in the $275 \mathrm{GHz}$ EPR spectra point to the presence of distinct populations of spin labels, which differ in the extent of hydrogen bonding. In Fig. 5a, the fractions of the populations, as obtained from the analysis of the $g_{x x}$ region of the $275 \mathrm{GHz}$ cw EPR spectrum, are represented as a function of $\left\langle g_{x x}\right\rangle$. The $\left\langle g_{x x}\right\rangle$ values have been obtained as the first moment of the EPR spectrum between $9.8000 \mathrm{~T}$ and $9.8150 \mathrm{~T}$. In this way we are independent of the Gaussian decomposition. Alternatively, we have calculated the $\left\langle g_{x x}\right\rangle$ value according to $\mathrm{fr}^{0} \cdot g_{x x}^{0}+\mathrm{fr}^{1} \cdot g_{x x}^{1}+\mathrm{fr}^{2}$. $g_{x x}^{2}$. These values are virtually identical ( $c f$. Table 1$)$, which underscores the quality of the Gaussian fits. Fig. 5a shows that the fraction of doubly hydrogen-bonded spin labels decreases with increasing $\left\langle g_{x x}\right\rangle$, the fraction of non-hydrogen-bonded spin labels increases with increasing $\left\langle g_{x x}\right\rangle$, and the fraction of singly hydrogen-bonded spin labels goes through a maximum. This course of the fractions reflects the shift of the protonation equilibria. Interestingly, the plot of the fractions as a function of $\left\langle A_{z z}\right\rangle$ in Fig. 5b shows large similarity with Fig. 5a. The increase of $\left\langle g_{x x}\right\rangle$ and the decrease of $\left\langle A_{z z}\right\rangle$ correlate similarly with the change in the composition, which supports the interpretation that both $g_{x x}$ and $A_{z z}$ are primarily determined by hydrogen bonding. The increase of $g_{x x}$ and the decrease of $A_{z z}$ going from two to one to zero hydrogen bonds are in line with theoretical predictions. ${ }^{19}$ In addition, not only the values of $g_{x x}^{2}$ but also of $\left\langle A_{z z}\right\rangle$ support the interpretation of population 2 as doubly hydrogen bonded spin labels. When $\mathrm{fr}^{2}$ is large, for br166 and br129 herein and for a number of other protein sites studied at $95 \mathrm{GHz},{ }^{2}$ the value of $\left\langle A_{z z}\right\rangle$ is larger than $3.63 \mathrm{mT}$. This value, observed in water, is the maximum value of $A_{z z}$ observed for singly hydrogen-bonded nitroxides, which means that doubly hydrogen-bonded spin labels have to be present to get an average value larger than $3.63 \mathrm{mT}$. In this analysis it remains an open question which potential hydrogen-bond donors qualify for the doubly hydrogen-bonded spin labels.
We summarize that the increase of $\left\langle g_{x x}\right\rangle$ and the decrease of $\left\langle A_{z z}\right\rangle$ follow the availability of proton donors, i.e., the proticity of the micro-environment at the spin-labeled protein site. This insight offers a description for the linear variation of $\left\langle g_{x x}\right\rangle$ with $\left\langle A_{z z}\right\rangle$ commonly derived from low-frequency cw EPR spectra. As shown in Fig. 6, the data for the spin-labeled protein sites at $275 \mathrm{GHz}$ also follow this behavior. The line that corresponds to the best linear fit of these data virtually coincides with the line that corresponds to the best linear fit to the data for the free nitroxide radical R1 in frozen solutions of (non-)hydrogenbonding solvents. The slope of the variation of the average parameter $\left\langle g_{x x}\right\rangle$ for the spin-labeled protein sites is much larger than the slope of $g_{x x}^{i}$ for the individual components. The small variation of each $g_{x x}^{i}$ reflects the small variation from one protein site to the other of the $g_{x x}$ value of the spin label carrying a definite number of hydrogen bonds. The large

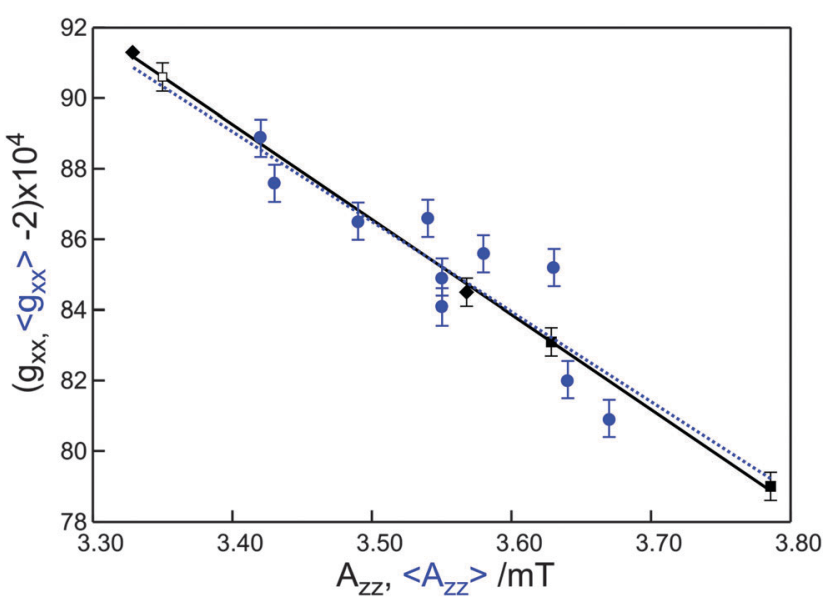

Fig. 6 Plot of $g_{x x}$ as a function of $A_{z z}$. The blue dots represent the average values $\left\langle g_{x x}\right\rangle$ and $\left\langle A_{z z}\right\rangle$ for the spin-labeled protein sites (cf. Table 1). The black dots represent values of $g_{x x}^{i}$ and $A_{z z}^{i}$ for the free nitroxide radical R1 in frozen water $(\boldsymbol{\square}, i=1,2), 2$-propanol $(\bullet, i=0,1){ }^{11}$ and orthoterphenyl/benzene $(\square, i=0){ }^{11}$ The dashed and solid lines with slopes of $-2.6 \pm 0.5 \mathrm{~T}^{-1}$ and $-2.69 \pm 0.05 \mathrm{~T}^{-1}$ represent the best linear fits to the experimental data for the spin-labeled protein sites and the nitroxide radical, respectively. 
variation of $\left\langle g_{x x}\right\rangle$ mostly reflects the variation in proticity from one protein site to the other.

\section{Conclusion}

Continuous-wave $275 \mathrm{GHz}$ EPR has provided the $g$-resolution necessary to achieve an unequivocal analysis of the $g_{x x}$ region of the spectrum of spin-labeled proteins in terms of the contributions of three distinct populations. For ten samples of spinlabeled membrane proteins we have determined the fraction of each population and the corresponding $g_{x x}$ value.

The populations correspond to spin labels that carry no, one and two hydrogen bonds. The $g_{x x}$ value of each population varies hardly from site to site, while the fractions of the different populations do. The change of $\left\langle A_{z z}\right\rangle$ from one to the other protein site follows the extent of hydrogen bonding. The $\left\langle g_{x x}\right\rangle$ and $\left\langle A_{z z}\right\rangle$ values both reflect the local proticity.

The cw EPR spectra present no clue as regards the nature of the hydrogen-bond donors. Both protein (amide and hydroxyl) and water protons qualify and it would be most relevant for a better functional understanding to identify the site-specific proton donors. In a recent study, a first attempt was made to disentangle the sources of protons involved in hydrogen bonding. ${ }^{3}$ Further progress requires the combination of $\mathrm{cw}$ and pulsed EPR studies with molecular-dynamics simulations.

\section{Acknowledgements}

R1 was kindly provided by Herbert Zimmermann (Max-Planck Institute for Medical Research, Heidelberg). The research was supported with financial aid by the Netherlands Organization for Scientific Research (NWO), project DN63-248 (A Dutch-German Cross-Border Initiative in Biosciences). Financial support by the Deutsch Forschungsgemeinschaft (DFG) is gratefully acknowledged (SFB 944; STE 640/6-2; MO 132/19-2), as is continued support by the Max Planck Gesellschaft and Freie Universität Berlin.

\section{References}

1 H. J. Steinhoff, A. Savitsky, C. Wegener, M. Pfeiffer, M. Plato and K. Möbius, Biochim. Biophys. Acta, 2000, 1457, 253.

2 E. Bordignon, H. Brutlach, L. Urban, K. Hideg, A. Savitsky, A. Schnegg, P. Gast, M. Engelhard, E. J. J. Groenen, K. Möbius and H. J. Steinhoff, Appl. Magn. Reson., 2010, 37, 391.

3 L. Urban and H. J. Steinhoff, Mol. Phys., 2013, 111, 2873.
4 M. A. Ondar, O. Y. Grinberg, A. A. Dubinskii, A. F. Shestakov and Y. S. Lebedev, Khim. Fiz., 1983, 2, 54; M. A. Ondar, O. Y. Grinberg, A. A. Dubinskii and Y. S. Lebedev, Khim. Fiz., 1984, 2, 527; O. Y. Grinberg, A. A. Dubinskii, V. N. Krymov, O. G. Poluektov and Y. S. Lebedev, Khim. Fiz., 1988, 7, 1011.

5 M. A. Ondar, O. Y. Grinberg, A. A. Dubinskii and Y. S. Lebedev, Sov. J. Chem. Phys., 1985, 3, 781.

6 J. S. Hwang, R. P. Mason, L.-P. Hwang and J. H. Freed, J. Phys. Chem., 1975, 79, 489.

7 R. Owenius, M. Engström, M. Lindgren and M. Huber, J. Phys. Chem. A, 2001, 105, 10967.

8 M. Plato, H. J. Steinhoff, C. Wegener, J. T. Törring, A. Savitsky and K. Möbius, Mol. Phys., 2002, 100, 3711.

9 Z. Ding, A. F. Gullá and D. E. Budil, J. Chem. Phys., 2001, 115, 10685.

10 A. Savitsky, A. A. Dubinskii, M. Plato, Y. S. Grishin, H. Zimmermann and K. Möbius, J. Phys. Chem. B, 2008, 112, 9079.

11 A. Nalepa, K. Möbius, W. Lubitz and A. Savitsky, J. Magn. Reson., 2014, 242, 203.

12 H. Brutlach, E. Bordignon, L. Urban, J. P. Klare, H.-J. Reyer, M. Engelhard and H. J. Steinhoff, Appl. Magn. Reson., 2006, 30, 359.

13 A. A. Wegener, I. Chizhov, M. Engelhard and H.-J. Steinhoff, J. Mol. Biol., 2000, 301, 881.

14 M. Pfeiffer, T. Rink, K. Gerwert, D. Oesterhelt and H.-J. Steinhoff, J. Mol. Biol., 1999, 287, 163.

15 H. Blok, J. A. J. M. Disselhorst, S. B. Orlinskii and J. Schmidt, J. Magn. Reson., 2004, 166, 92; H. Blok, J. A. J. M. Disselhorst, H. van der Meer, S. B. Orlinskii and J. Schmidt, J. Magn. Reson., 2005, 173, 49; G. Mathies, H. Blok, J. A. J. M. Disselhorst, P. Gast, H. van der Meer, D. M. Miedema, R. M. Almeida, J. J. G. Moura, W. R. Hagen and E. J. J. Groenen, J. Magn. Reson., 2011, 210, 126-132.

16 K. Möbius, A. Savitsky, A. Schnegg, M. Plato and M. Fuchs, Phys. Chem. Chem. Phys., 2005, 7, 19; K. Möbius and A. Savitsky, High-field EPR spectroscopy on proteins and their model systems, RSC Publishing, Cambridge, UK, 2009, p. 375 .

17 H.-J. Steinhoff, N. Radzwill, W. Thevis, V. Lenz, D. Brandenburg, A. Antson, G. Dodson and A. Wollmer, Biophys. J., 1997, 73, 3287.

18 M. Pavone, P. Cimino, O. Crescenzi, A. Sillanpää and V. Barone, J. Phys. Chem. B, 2007, 111, 8928.

19 M. Pavone, A. Sillanpää, P. Cimino, O. Crescenzi and V. Barone, J. Phys. Chem. B, 2006, 110, 16189. 